\title{
Practical stability of stochastic delay evolution equations
}

\author{
Tomás Caraballo $^{a} \quad$ Mohamed Ali Hammami $^{b} \quad$ Lassad Mchiri $^{b}$ \\ ${ }^{a}$ Universidad de Sevilla, \\ Dpto. Ecuaciones Diferenciales y Análisis Numérico, \\ Facultad de Matemáticas, Sevilla (Spain) \\ ${ }^{b}$ University of Sfax, Faculty of Sciences of Sfax, Department of Mathematics, Tunisia \\ E.mail: MohamedAli.Hammami@fss.rnu.tn
}

\begin{abstract}
In this paper we investigate the almost sure practical stability for a class of stochastic functional evolution equations. We establish some sufficient conditions based on the construction of appropriate Lyapunov functional. The abstract results are then applied to some illustrative examples.
\end{abstract}

Keywords: Stochastic delay evolution equations, almost sure practical asymptotic stability, decay function.

MSC: $60 \mathrm{H} 15$,

\section{Introduction}

Stochastic differential equations are useful for modeling physical, technical, biological and economical dynamical systems in which significant uncertainty is present. In 1892, Lyapunov introduced the concept of stability for deterministic dynamical systems and firstly established the so-called Lyapunov second method of stability theory. The advantage of this method can judged the stability of systems without knowledge of the solution of the systems explicitly. It is therefore of great interest to be able to characterize the behavior of the solutions. Several interesting and important variants to Lyapunov's original concepts of stability were proposed in [1]-[3] and [14]. However, when the origin is not necessarily an equilibrium point, it is still possible to analyze the asymptotic stability of solutions with respect to a small neighborhood of the origin, what yields 
to the concept of practical stability. It is worth mentioning some previous works on practical stability in the deterministic framework, as for example, [1], [3]. Also we would like to mention here the references $[4,5,19,20]$, among others. As for the stochastic case, we initiate the study of this practical stability for non-delay stochastic partial differential equations in [14].

Needless to say that the asymptotic behavior of systems described by stochastic evolution equations is a very important topic as the vast literature on this field shows (see $[7,8,9,10$, $11,12,13,16,19,20,22,23,25]$. Moreover, the consideration of memory terms in the models are fully justified as it is sensible to think that not only the present state of the systems possesses influence in the future behavior of the problem, but also what has happened in the past. Additionally, in the nonlinear and/or nonautonomous situations, it may happen that the stability cannot always be exponential but can even be sub or super-exponential (see [12]). For this reason, in this paper we will study the almost sure practical stability with general decay rate and will illustrate the theory with some application examples.

The content of the paper is as follows. In section 2, we introduce the basic notations and assumptions. In Section 3, we prove some sufficient conditions ensuring almost sure practical stability of solutions to stochastic delay evolution equations. First we prove a sufficient condition ensuring the convergence of solutions to zero with general decay rate, even in the case that zero is not an equilibrium point. Later, we weaken the assumptions on the operators involved in the model, obtaining a weaker result which will ensure the convergence to a ball with a small radius, instead of the convergence of solutions to zero. In Section 4, we finally study two examples to illustrate these results.

\section{Basic notations and assumptions}

Let $V$ be a reflexible Banach space and $H$ a real separable Hilbert space such that

$$
V \subset H \equiv H^{*} \subset V^{*}
$$

where the injections are continuous and dense. In addition, we also assume both $V$ and $V^{*}$ are uniformly convex.

We denote by $\|\|,.|$.$| and \|.\|_{*}$ the norms in $V, H$ and $V^{*}$ respectively, by (.,.) the inner product in $H$, and by $<., .>$ the duality between $V$ and $V^{*}$.

Assume $\{\Omega, \mathcal{F}, \mathbb{P}\}$ is a complete probability space with a normal filtration $\left\{\mathcal{F}_{t}\right\}_{t \geq 0}$, i.e. $\mathcal{F}_{0}$ contains the null sets in $\mathcal{F}$ and $\mathcal{F}_{t}=\cap_{s>t} \mathcal{F}_{s}$, for all $t \geq 0$, and let us consider a real valued $\left\{\mathcal{F}_{t}\right\}$-Wiener process $\{W(t)\}_{t \geq 0}$.

Given $h \geq 0, p \geq 2$ and $T>0$, we denote by $I^{p}(-h, T, V)$ the closed subspace of $L^{p}(\Omega \times(-h, T), \mathcal{F} \otimes \mathcal{B}([-h, T]), \mathcal{P} \otimes d t ; V)$ of all stochastic processes which are $\mathcal{F}_{t^{-}}$adapted 
for almost every $t$ in $(0, T)$ (in what follows, a.e.t), where $\mathcal{B}([0, T])$ denotes the Borel $\sigma$-algebra of subsets in $[0, T]$ and we will set $\mathcal{F}_{t}=\mathcal{F}_{0}$ for $t<0$. We write $L^{2}(\Omega ; C(-h, T ; H))$ instead of $L^{2}(\Omega, \mathcal{F}, \mathcal{P} ; C(-h, T ; H))$, where $C(-h, T ; H)$ denotes the space of all continuous functions from $[-h, T]$ into $H$.

Let $C_{H}=C(-h, 0 ; H)$ with norm $|\psi|_{C_{H}}=\sup _{-h \leq s \leq 0}|\psi(s)|, \psi \in C_{H} ; L_{V}^{p}=L^{p}([-h, 0], V)$ and $L_{H}^{p}=L^{p}([-h, 0], H)$.

On the other hand, given a stochastic process

$$
X(t) \in I^{p}(-h, T, V) \cap L^{2}(\Omega ; C(-h, T ; H)),
$$

we associate with another stochastic process

$$
X_{t}: \Omega \rightarrow L_{V}^{p} \cap C_{H},
$$

by means of the usual relation $X_{t}(s)(\omega)=X(t+s)(\omega), 0 \leq t \leq T,-h \leq s \leq 0$.

Let us consider the following stochastic delay evolution equation in $V^{*}$ :

$$
\left\{\begin{aligned}
d X(t) & =\left(A(t, X(t))+F\left(t, X_{t}\right)\right) d t+G\left(t, X_{t}\right) d W_{t}, \quad t \in[0, T] \\
X(t) & =\psi(t), \quad t \in[-h, 0]
\end{aligned}\right.
$$

where $T>0$ and the initial datum $\psi \in I^{p}(-h, 0, V) \cap L^{2}\left(\Omega ; C_{H}\right)$.

As we are mainly interested in the stability analysis of solutions to equation (2.1), we shall assume that for each $\psi \in I^{p}(-h, 0, V) \cap L^{2}\left(\Omega ; C_{H}\right)$ there exists a process

$$
X(t) \in I^{p}(-h, T, V) \cap L^{2}(\Omega ; C(-h, T ; H)),
$$

which is solution to equation (2.1) for every $T>0$, in other words, $X(t)$ satisfies the following integral equation in $V^{*}$ :

$$
\left\{\begin{array}{l}
X(t)=\psi(0)+\int_{0}^{t}\left(A(s, X(s))+F\left(s, X_{s}\right)\right) d s+\int_{0}^{t} G\left(s, X_{s}\right) d W_{s}, \quad \forall t \in[0, T] \\
X(t)=\psi(t), \quad t \in[-h, 0] .
\end{array}\right.
$$

This happens, for instance, if $A(t,):. V \rightarrow V^{*}$ is a family of (nonlinear) operators defined a.e.t. satisfying there exists $t \in \mathbb{R}_{+}$such that $A(t, 0) \neq 0$ and fulfilling the following assumptions :

1. Measurability: for every $x \in V$, the map $t \in(0, T) \mapsto A(t, x) \in V^{*}$ is Lebesgue measurable, a.e. $t$.

2. Hemicontinuity: The map $\theta \in \mathbb{R} \mapsto<A(t, x+\theta y), z>\in \mathbb{R}$ is continuous for every $x, y, z \in V$, a.e. $t$.

3. Boundedness: There exists $\beta>0, c>0$ such that

$$
\|A(t, x)\|_{*} \leq c\|x\|^{p-1}+\beta \quad \text { for all } x \in V, \quad \text { a.e.t. }
$$


4. Coercivity: There exist $\alpha>0, \lambda, \gamma \in \mathbb{R}$ such that

$$
-2<A(t, x), x>+\lambda|x|^{2}+\gamma \geq \alpha\|x\|^{p} \quad \text { for all } x \in V, \quad \text { a.e.t. }
$$

5. Monotonicity:

$$
\lambda|x-y|^{2}-(2<A(t, x)-A(t, y), x-y>) \geq 0 \quad \text { for all } x, y \in V, \quad \text { a.e.t. }
$$

Also $F(t,):.[0, T] \times C_{H} \rightarrow V^{*}$ is a family of Lipschitz continuous operators defined a.e.t. and $G(t,):.[0, T] \times C_{H} \rightarrow H$ is another family of Lipschitz operators defined a.e.t. (see [7], [8] and also [24]) and satisfying there exists $t \in \mathbb{R}_{+}$such that $G(t, 0) \neq 0$.

Unless otherwise is stated, we will assume that $U(t, x)$ is a $C^{1,2}$-positive appropriate Lyapunov functional such that for any $x \in V$ and $t \in \mathbb{R}_{+}, U_{x}^{\prime}(t, x) \in V$, and satisfies some additional assumptions which enable us to apply the Itô formula for the process $X(t)$, solution to equation (2.1) (see [21]).

\section{Practical stability of stochastic delay evolution equa- tion}

We first state the definition of almost surely convergence of solutions to a small ball centered at the origin.

Definition 3.1. The ball $B_{r}:=\{x \in H /|x| \leq r\}, r>0$ is said to be almost surely globally practically uniformly exponentially stable with respect to the system (2.1) if:

For any initial value $\psi \in I^{p}(-h, 0, V) \cap L^{2}\left(\Omega ; C_{H}\right)$ such that $0<|X(t, \psi)|-r$, for all $t \geq t_{0} \geq 0$, $\mathbb{P}$-a.s., where $X(t, \psi)$ denotes the solution of $(2.1)$, it holds that

$$
\lim _{t \rightarrow \infty} \sup \frac{1}{t} \ln (|X(t, \psi)|-r)<0, \mathbb{P}-\text { a.s. }
$$

System (2.1) is said to be almost surely globally practically uniformly exponentially stable if there exists $r>0$ such that $B_{r}$ is almost surely globally practically uniformly exponentially stable.

Next we state the definition of practical convergence to zero with general decay rate.

Definition 3.2. Let $\lambda(t)$ be a positive function defined for sufficiently large $t>0$, say $t \geq T>0$, and satisfying that $\lambda(t) \uparrow+\infty$ as $t \rightarrow+\infty$. A solution $X(t)$ to equation (2.1) (defined in the future, i.e. for $t$ large enough) is said to decay to zero almost surely with decay function $\lambda(t)$ and order at least $\gamma>0$, if there exists a function $r(\cdot)$ such that $r(t) \rightarrow 0$ as $t \rightarrow+\infty$, and its generalized Lyapunov exponent is less than or equal to $-\gamma$ with probability one, i.e.

$$
\lim _{t \rightarrow+\infty} \sup \frac{\ln (|X(t)|-r(t))}{\ln (\lambda(t))} \leq-\gamma, \quad \mathbb{P}-\text { a.s. }
$$


If in addition 0 is solution to equation (2.1), the zero solution is said to be almost surely practically asymptotically stable with decay function $\lambda(t)$ and order at least $\gamma$, if there exist $r(t) \rightarrow 0$ as $t \rightarrow+\infty$ such that every solution to equation (2.1) decays to zero almost surely with decay function $\lambda(t)$ and order at least $\gamma$.

Now, we can state and prove one of our main results in this paper.

Theorem 3.1. Let $U(t, x)$ be an appropriate Lyapunov functional. Assume that $\ln \lambda(t)$ is uniformly continuous on $t \in[T,+\infty)$ and there exists a constant $\tau \geq 0$ such that

$$
\lim _{t \rightarrow \infty} \sup \frac{\ln \ln (t)}{\ln \lambda(t)} \leq \tau .
$$

Assume that there exist constants $q \in \mathbb{N}^{*}, m>0, \mu \geq 0, b \geq 0, \nu, \theta \geq 0$, a non-increasing function $\xi(t)>0$ and a continuous non-negative function $\varphi(t)$ such that

$$
(a)|x|^{q} \lambda(t)^{m} \leq U(t, x), \quad \forall(t, x) \in \mathbb{R}_{+} \times V .
$$

(b) For a solution $X(t)$ to (2.1), defined in the future, it holds

$$
\begin{aligned}
\int_{0}^{t} U_{s}^{\prime}(s, X(s)) d s & +\int_{0}^{t}<U_{x}^{\prime}(s, X(s)), A(s, X(s))+F\left(s, X_{s}\right)>d s \\
& +\frac{1}{2} \int_{0}^{t}\left(U_{x x}^{\prime \prime}(s, X(s)) G\left(s, X_{s}\right), G\left(s, X_{s}\right)\right) d s \\
& +\int_{0}^{t} \xi(s)\left(U_{x}^{\prime}(s, X(s)), G\left(s, X_{s}\right)\right)^{2} d s \\
& \leq \zeta_{\psi}(t)+\varrho(t)+\int_{0}^{t} \varphi(s) U(s, X(s)) d s
\end{aligned}
$$

where $\zeta_{\psi}(t)$ is a non-negative function depending on the initial datum $\psi$ and $\varrho(t) \neq 0$, for all $t \geq 0$, is a function independent on $\psi$ such that $\lim _{t \rightarrow \infty} \varrho(t)=0$.

$$
\begin{aligned}
& \text { (c) } \lim _{t \rightarrow \infty} \sup \frac{\ln \zeta_{\psi}(t)}{\ln \lambda(t)} \leq \nu, \quad \lim _{t \rightarrow \infty} \sup \frac{\int_{0}^{t} \varphi(s) d s}{\ln \lambda(t)} \leq \theta . \\
& \lim _{t \rightarrow \infty} \sup \frac{\ln \left(c+\frac{\varrho(t)}{\lambda(t)^{\delta}}\right)}{\ln \lambda(t)} \leq b, \quad \lim _{t \rightarrow \infty} \inf \frac{\ln \xi(t)}{\ln \lambda(t)} \geq-\mu .
\end{aligned}
$$

where $\delta=(\mu+\tau+2 \epsilon) \vee(\nu+\epsilon)$ for arbitrary $\epsilon>0$ and

$$
\left\{\begin{array}{l}
c=\exp (\epsilon(\mu+\epsilon)), \quad \text { if } \quad \delta=\mu+\tau+2 \epsilon \\
c=1, \quad \text { if } \delta=\nu+\epsilon
\end{array}\right.
$$




$$
(d) \sum_{k=1}^{q}|x|^{q-k}\left[\frac{|\varrho(t)|}{\lambda(t)^{m}}\right]^{\frac{k-1}{q}} \geq 1, \quad \text { for all } t \geq 0
$$

Then,

$$
\lim _{t \rightarrow+\infty} \sup \frac{\ln \left(|X(t)|-\left(\frac{|\varrho(t)|}{\lambda(t)^{m}}\right)^{\frac{1}{q}}\right)}{\ln \lambda(t)} \leq-[m-(\theta+b+((\mu+\tau) \vee \nu))], \quad \mathbb{P}-\text { a.s. }
$$

In particular, if $m>\theta+b+((\mu+\tau) \vee \nu)$, the solution $X(t)$ decays to zero almost surely with decay function $\lambda(t)$ and order at least

$$
\gamma=[m-(\theta+b+((\mu+\tau) \vee \nu))] .
$$

Proof. Assume that $\lambda(t)^{m}|x|^{q}-|\varrho(t)|>0$ for all $t \geq 0$ and $x \in H$. Then, we immediately have $\lambda(t)^{m}|x|^{q}-|\varrho(t)| \leq \lambda(t)^{m}|x|^{q} \leq U(t, x)$. Now, observe that

$$
\begin{aligned}
\lambda(t)^{m}|x|^{q}-|\varrho(t)| & =\lambda(t)^{m}\left(|x|^{q}-\frac{|\varrho(t)|}{\lambda(t)^{m}}\right) \\
& =\lambda(t)^{m}\left(|x|^{q}-\left(\left(\frac{\varrho(t) \mid}{\lambda(t)^{m}}\right)^{\frac{1}{q}}\right)^{q}\right) \\
& =\lambda(t)^{m}\left(|x|-\left(\frac{|\varrho(t)|}{\lambda(t)^{m}}\right)^{\frac{1}{q}}\right)\left(|x|^{q-1}+|x|^{q-2}\left(\frac{\varrho(t) \mid}{\lambda(t)^{m}}\right)^{\frac{1}{q}}+\ldots+\left(\frac{\varrho \varrho(t) \mid}{\lambda(t)^{m}}\right)^{\frac{q-1}{q}}\right) \\
& =\lambda(t)^{m}\left(|x|-\left(\frac{|\varrho(t)|}{\lambda(t)^{m}}\right)^{\frac{1}{q}}\right) \sum_{k=1}^{q}|x|^{q-k}\left[\frac{|\varrho(t)|}{\lambda(t)^{m}}\right]^{\frac{k-1}{q}} \\
& \geq \lambda(t)^{m}\left(|x|-\left(\frac{|\varrho(t)|}{\lambda(t)^{m}}\right)^{\frac{1}{q}}\right) .
\end{aligned}
$$

Therefore, $\lambda(t)^{m}\left(|x|-\left(\frac{\varrho(t) \mid}{\lambda(t)^{m}}\right)^{\frac{1}{q}}\right) \leq U(t, x)$ and $\ln \left[\lambda(t)^{m}\left(|x|-\left(\frac{|\varrho(t)|}{\lambda(t)^{m}}\right)^{\frac{1}{q}}\right)\right] \leq \ln (U(t, x))$. Thus $\ln \left[\left(|x|-\left(\frac{|\varrho(t)|}{\lambda(t)^{m}}\right)^{\frac{1}{q}}\right)\right]+m \ln (\lambda(t)) \leq \ln (U(t, x))$.

Applying the Itô formula we obtain

$$
\begin{aligned}
U(t, X(t))= & U(0, \psi(0))+\int_{0}^{t} U_{s}^{\prime}(s, X(s)) d s \\
& +\int_{0}^{t}<U_{x}^{\prime}(s, X(s)), A(s, X(s))+F\left(s, X_{s}\right)>d s \\
& +\frac{1}{2} \int_{0}^{t}\left(U_{x x}^{\prime \prime}(s, X(s)) G\left(s, X_{s}\right), G\left(s, X_{s}\right)\right) d s \\
& +\int_{0}^{t}\left(U_{x}^{\prime}(s, X(s)), G\left(s, X_{s}\right)\right) d W(s) .
\end{aligned}
$$


Now, from the uniform continuity of $\ln \lambda(t)$, we can ensure that for each $\epsilon>0$ there exist two positive integers $N=N(\epsilon)$ and $k_{1}(\epsilon)$ such that if $\frac{k-1}{2^{N}} \leq t \leq \frac{k}{2^{N}}, k \geq k_{1}(\epsilon)$, it follows

$$
\left|\ln \lambda\left(\frac{k}{2^{N}}\right)-\ln \lambda(t)\right| \leq \epsilon .
$$

On the other hand, due to the exponential martingale inequality

$$
\mathbb{P}\left\{\omega ; \sup _{0 \leq t \leq w}\left[M(t)-\frac{u}{2} \int_{0}^{t}\left(U_{x}^{\prime}(s, X(s)), G\left(s, X_{s}\right)\right)^{2} d s\right]>\sigma\right\} \leq e^{-u \nu},
$$

for any positive constants $u, \sigma$ and $w$, where

$$
M(t)=\int_{0}^{t}\left(U_{x}^{\prime}(s, X(s)), G\left(s, X_{s}\right)\right) d W(s) .
$$

In particular, for the preceding $\epsilon>0$, if we set $u=2 \xi\left(\frac{k}{2^{N}}\right), \sigma=\xi\left(\frac{k}{2^{N}}\right)^{-1} \ln \frac{k-1}{2^{N}}, w=\frac{k}{2^{N}}$, $k=2,3, \ldots$, we can then apply the Borel-Cantelli lemma to obtain that, for almost all $\omega \in \Omega$, there exists an integer $k_{0}(\epsilon, \omega)>0$ such that

$$
\begin{aligned}
\int_{0}^{t}\left(U_{x}^{\prime}(s, X(s)), G\left(s, X_{s}\right)\right) d W(s) & \leq \xi\left(\frac{k}{2^{N}}\right)^{-1} \ln \frac{k-1}{2^{N}} \\
& +\xi\left(\frac{k}{2^{N}}\right) \int_{0}^{t}\left(U_{x}^{\prime}(s, X(s)), G\left(s, X_{s}\right)\right)^{2} d s \\
& \leq \xi\left(\frac{k}{2^{N}}\right)^{-1} \ln \frac{k-1}{2^{N}} \\
& +\int_{0}^{t} \xi(s)\left(U_{x}^{\prime}(s, X(s)), G\left(s, X_{s}\right)\right)^{2} d s,
\end{aligned}
$$

for $0 \leq t \leq \frac{k}{2^{N}}, k \geq k_{0}(\epsilon, \omega)$. Replacing this into equation (3.3), we see that $\mathbb{P}$-a.s.

$$
\begin{aligned}
U(t, X(t)) \leq & U(0, \psi(0))+\xi\left(\frac{k}{2^{N}}\right)^{-1} \ln \frac{k-1}{2^{N}}+\int_{0}^{t} U_{s}^{\prime}(s, X(s)) d s \\
& +\int_{0}^{t}<U_{x}^{\prime}(s, X(s)), A(s, X(s))+F\left(s, X_{s}\right)>d s \\
& +\frac{1}{2} \int_{0}^{t}\left(U_{x x}^{\prime \prime}(s, X(s)) G\left(s, X_{s}\right), G\left(s, X_{s}\right)\right) d s \\
& +\int_{0}^{t} \xi(s)\left(U_{x}^{\prime}(s, X(s)), G\left(s, X_{s}\right)\right)^{2} d s,
\end{aligned}
$$


for $0 \leq t \leq \frac{k}{2^{N}}, k \geq k_{0}(\epsilon, \omega)$. Using condition (b) we derive that $\mathbb{P}$-a.s.

$$
\begin{aligned}
U(t, X(t)) \leq U & (0, \psi(0))+\xi\left(\frac{k}{2^{N}}\right)^{-1} \ln \frac{k-1}{2^{N}}+\zeta_{\psi}(t)+\varrho(t), \\
& +\int_{0}^{t} \varphi(s) U(s, X(s)) d s
\end{aligned}
$$

for $0 \leq t \leq \frac{k}{2^{N}}, k \geq k_{0}(\epsilon, \omega)$. By virtue of the Gronwall lemma we derive that $\mathbb{P}$-a.s.

$$
\begin{aligned}
U(t, X(t)) & \leq\left(U(0, \psi(0))+\xi\left(\frac{k}{2^{N}}\right)^{-1} \ln \frac{k-1}{2^{N}}+\zeta_{\psi}(t)+\varrho(t)\right) \\
& \times \exp \left(\int_{0}^{t} \varphi(s) d s\right),
\end{aligned}
$$

for $0 \leq t \leq \frac{k}{2^{N}}, k \geq k_{0}(\epsilon, \omega)$. On the other hand, thanks to condition (c) and the uniform continuity of $\ln \lambda(t)$ there exists a positive integer $k_{1}(\epsilon)$ such that whenever $t \geq k_{1}(\epsilon)$

$$
\zeta_{\psi}(t) \leq \lambda(t)^{\nu+\epsilon}, \quad \int_{0}^{t} \varphi(s) d s \leq(\theta+\epsilon) \ln \lambda(t), \quad \xi\left(\frac{k}{2^{N}}\right)^{-1} \leq e^{\epsilon(\mu+\epsilon)} \lambda(t)^{\mu+\epsilon},
$$

for $\frac{k-1}{2^{N}} \leq t \leq \frac{k}{2^{N}}, k \geq k_{1}(\epsilon)$. Also

$$
\ln \frac{k-1}{2^{N}} \leq \ln t \leq \ln \frac{k}{2^{N}} \quad \text { for } \quad \frac{k-1}{2^{N}} \leq t \leq \frac{k}{2^{N}} .
$$

We therefore obtain that, for almost all $\omega \in \Omega$,

$$
\begin{aligned}
\ln U(t, X(t)) \leq \ln & \left(U(0, \psi(0))+\lambda(t)^{\mu+\tau+2 \epsilon} e^{\epsilon(\mu+\epsilon)}+\lambda(t)^{\nu+\epsilon}+\varrho(t)\right) \\
& +(\theta+\epsilon) \ln \lambda(t),
\end{aligned}
$$

for $\frac{k-1}{2^{N}} \leq t \leq \frac{k}{2^{N}}, k \geq k_{1}(\epsilon)$. Hence

$$
\lim _{t \rightarrow+\infty} \sup \frac{\ln U(t, X(t))}{\ln \lambda(t)} \leq \delta+\theta+\epsilon+b, \quad \mathbb{P}-a . s .
$$

Taking into account that $\epsilon>0$ is arbitrary and using (a) and

$$
\ln \left[\left(|x|-\left(\frac{|\varrho(t)|}{\lambda(t)^{m}}\right)^{\frac{1}{q}}\right)\right]+m \ln (\lambda(t)) \leq \ln (U(t, x))
$$


we can deduce

$$
\lim _{t \rightarrow+\infty} \sup \frac{\ln \left(|X(t)|-\left(\frac{|\varrho(t)|}{\lambda(t)^{m}}\right)^{\frac{1}{q}}\right)}{\ln \lambda(t)} \leq-[m-(\theta+b+((\mu+\tau) \vee \nu))], \quad \mathbb{P}-\text { a.s. }
$$

as required.

Remark 3.2. In the next theorem we will weaken the assumptions imposed on the operators in our model and, as a counterpart, we will obtain a weaker convergence too. In fact, instead of obtaining practical convergence to zero with general decay rate, we will deduce only practical convergence to a ball.

Theorem 3.3. Let $U(t, x)$ be an appropriate Lyapunov functional. Assume that $\ln \lambda(t)$ is uniformly continuous on $t \in[T,+\infty)$ and there exists a constant $\tau \geq 0$ such that

$$
\lim _{t \rightarrow \infty} \sup \frac{\ln \ln (t)}{\ln \lambda(t)} \leq \tau
$$

Assume that there exist constants $q \in \mathbb{N}^{*}, m>0, \mu \geq 0, b>0, \nu, \theta \geq 0$ and a small constant $\varrho>0$, a non-increasing function $\xi(t)>0$ and a continuous non-negative function $\varphi(t)$ such that

$$
(a)|x|^{q} \lambda(t)^{m} \leq U(t, x), \quad \forall(t, x) \in \mathbb{R}_{+} \times V .
$$

(b) For a solution $X(t)$ to defined in the future it holds equation (2.1)

$$
\begin{aligned}
\int_{0}^{t} U_{s}^{\prime}(s, X(s)) d s & +\int_{0}^{t}<U_{x}^{\prime}(s, X(s)), A(s, X(s))+F\left(s, X_{s}\right)>d s \\
& +\frac{1}{2} \int_{0}^{t}\left(U_{x x}^{\prime \prime}(s, X(s)) G\left(s, X_{s}\right), G\left(s, X_{s}\right)\right) d s \\
& +\int_{0}^{t} \xi(s)\left(U_{x}^{\prime}(s, X(s)), G\left(s, X_{s}\right)\right)^{2} d s \\
& \leq \zeta_{\psi}(t)+\varrho+\int_{0}^{t} \varphi(s) U(s, X(s)) d s
\end{aligned}
$$

where $\zeta_{\psi}(t)$ is a non-negative function depending on the initial datum $\psi$.

$$
\begin{gathered}
\text { (c) } \lim _{t \rightarrow \infty} \sup \frac{\ln \zeta_{\psi}(t)}{\ln \lambda(t)} \leq \nu, \quad \lim _{t \rightarrow \infty} \sup \frac{\int_{0}^{t} \varphi(s) d s}{\ln \lambda(t)} \leq \theta, \\
\lim _{t \rightarrow \infty} \inf \frac{\ln \xi(t)}{\ln \lambda(t)} \geq-\mu . \\
(d) \lambda(t) \geq b ; \quad \sum_{k=1}^{q}|x|^{q-k}\left[\frac{\varrho}{b^{m}}\right]^{\frac{k-1}{q}} \geq 1, \quad \text { for all } t \geq 0 .
\end{gathered}
$$


Then,

$$
\lim _{t \rightarrow+\infty} \sup \frac{\ln \left(|X(t)|-\left(\frac{\varrho}{b^{m}}\right)^{\frac{1}{q}}\right)}{\ln \lambda(t)} \leq-[m-(\theta+((\mu+\tau) \vee \nu))], \quad \mathbb{P}-\text { a.s. }
$$

In particular, if $m>\theta+((\mu+\tau) \vee \nu)$ and $\lambda(t)=e^{t}$, the ball $B_{r}$ with $r=\left(\frac{\varrho}{b^{m}}\right)^{\frac{1}{q}}$ is almost surely globally practically uniformly exponentially stable.

Proof. Assuming that $b^{m}|x|^{q}-\varrho>0$, for all $x \in H$, then $b^{m}|x|^{q}-\varrho \leq \lambda(t)^{m}|x|^{q} \leq U(t, x)$. Now, observe that

$$
\begin{aligned}
b^{m}|x|^{q}-\varrho & =b^{m}\left(|x|^{q}-\frac{\varrho}{b^{m}}\right) \\
& =b^{m}\left(|x|^{q}-\left(\left(\frac{\varrho}{b^{m}}\right)^{\frac{1}{q}}\right)^{q}\right) \\
& =b^{m}\left(|x|-\left(\frac{\varrho}{b^{m}}\right)^{\frac{1}{q}}\right)\left(|x|^{q-1}+|x|^{q-2}\left(\frac{\varrho}{b^{m}}\right)^{\frac{1}{q}}+\ldots+\left(\frac{\varrho}{b^{m}}\right)^{\frac{q-1}{q}}\right) \\
& =b^{m}\left(|x|-\left(\frac{\varrho}{b^{m}}\right)^{\frac{1}{q}}\right) \sum_{k=1}^{q}|x|^{q-k}\left[\frac{\varrho}{b^{m}}\right]^{\frac{k-1}{q}} \\
& \geq b^{m}\left(|x|-\left(\frac{\varrho}{b^{m}}\right)^{\frac{1}{q}}\right) .
\end{aligned}
$$

According to the hypothesis that $\lambda(t) \geq b$, for all $t \geq 0$ and $b^{m}|x|^{q}-\varrho>0$, we can observe that

$$
\lambda(t)^{m}\left(|x|-\left(\frac{\varrho}{b^{m}}\right)^{\frac{1}{q}}\right) \leq \lambda(t)^{m}|x|^{q} \leq U(t, x) .
$$

Therefore, $\ln \left[\lambda(t)^{m}\left(|x|-\left(\frac{\varrho}{b^{m}}\right)^{\frac{1}{q}}\right)\right] \leq \ln (U(t, x))$ and $\ln \left[\left(|x|-\left(\frac{\varrho}{b^{m}}\right)^{\frac{1}{q}}\right)\right]+m \ln (\lambda(t)) \leq \ln (U(t, x))$. Applying once more the Itô formula,

$$
\begin{aligned}
U(t, X(t)) & =U(0, \psi(0))+\int_{0}^{t} U_{s}^{\prime}(s, X(s)) d s \\
& +\int_{0}^{t}<U_{x}^{\prime}(s, X(s)), A(s, X(s))+F\left(s, X_{s}\right)>d s \\
& +\frac{1}{2} \int_{0}^{t}\left(U_{x x}^{\prime \prime}(s, X(s)) G\left(s, X_{s}\right), G\left(s, X_{s}\right)\right) d s \\
& +\int_{0}^{t}\left(U_{x}^{\prime}(s, X(s)), G\left(s, X_{s}\right)\right) d W(s) .
\end{aligned}
$$

Proceeding as in the proof of Theorem 3.1, We therefore obtain that for almost all $\omega \in \Omega$

$$
\begin{aligned}
\ln U(t, X(t)) & \leq \ln \left(U(0, \psi(0))+\lambda(t)^{\mu+\tau+2 \epsilon} e^{\epsilon(\mu+\epsilon)}+\lambda(t)^{\nu+\epsilon}+\varrho\right) \\
& +(\theta+\epsilon) \ln \lambda(t),
\end{aligned}
$$


for $\frac{k-1}{2^{N}} \leq t \leq \frac{k}{2^{N}}, k \geq k_{1}(\epsilon)$. Hence

$$
\lim _{t \rightarrow+\infty} \sup \frac{\ln U(t, X(t))}{\ln \lambda(t)} \leq(\mu+\tau+2 \epsilon) \vee(\nu+\epsilon)+\theta+\epsilon, \quad \mathbb{P}-\text { a.s. }
$$

Taking into account that $\epsilon>0$ is arbitrary and using (a) and

$$
\ln \left[\left(|x|-\left(\frac{\varrho}{b^{m}}\right)^{\frac{1}{q}}\right)\right]+m \ln (\lambda(t)) \leq \ln (U(t, x)),
$$

we can deduce

$$
\lim _{t \rightarrow+\infty} \sup \frac{\ln \left(|X(t)|-\left(\frac{\varrho}{b^{m}}\right)^{\frac{1}{q}}\right)}{\ln \lambda(t)} \leq-[m-(\theta+((\mu+\tau) \vee \nu))], \quad \mathbb{P}-\text { a.s. }
$$

as required.

Remark 3.4. Notice that the hypothesis $\lambda(t) \geq b$ can be omitted in assumption $(d)$ since $\lambda(t) \rightarrow+\infty$ as $t \rightarrow+\infty$.

\section{Examples}

Now, we illustrate our abstract results with two examples.

Example 4.1. We consider the following one dimensional model with constant time delay

$$
\left\{\begin{aligned}
d X(t) & =\left[-\frac{q-1}{1+t} X(t)+\frac{1}{1+t} X(t-h)\right] d t+(1+t)^{-q} d W_{t}, \quad t \in[0, T], \\
X(t) & =\psi(t), \quad t \in[-h, 0]
\end{aligned}\right.
$$

where $q>2$ and $T, h>0$ and $|\psi|_{C} \geq 1$. This problem can be set in our formulation by taking $V=H=\mathbb{R}, p=2$. We will write $C$ instead of $C_{H}$. From the standard theory on stochastic differential equations with delays, it is straightforward that the preceding problem possesses a unique solution for each initial datum fixed in the space $I^{2}(-h, 0 ; \mathbb{R}) \cap L^{2}(\Omega ; C)$. Define for $u \in \mathbb{R}$ and $\phi \in C, A(t, u)=-\frac{(q-1) u}{1+t}, F(t, \phi)=\frac{1}{1+t} \phi(-h)$ and $G(t, \phi)=(1+t)^{-q}, t \in[0, T]$. Now we consider $U(t, y)=(1+t)^{2 q-2}|y|^{2}$. Then, it is easy to check that for arbitrary $\sigma>1$, 


$$
\begin{aligned}
& \xi(t)=\frac{1}{4(1+t)^{\sigma}} \text {, we have } \\
& \int_{0}^{t} U_{s}^{\prime}(s, X(s)) d s+\int_{0}^{t}<U_{x}^{\prime}(s, X(s)), A(s, X(s))+F\left(s, X_{s}\right)>d s \\
& +\frac{1}{2} \int_{0}^{t}\left(U_{x x}^{\prime \prime}(s, X(s)) G\left(s, X_{s}\right), G\left(s, X_{s}\right)\right) d s \\
& +\int_{0}^{t} \frac{1}{4(1+s)^{\sigma}}\left(U_{x}^{\prime}(s, X(s)), G\left(s, X_{s}\right)\right)^{2} d s \\
& \leq \int_{0}^{t} \frac{1}{(1+s)^{2}} d s+\int_{0}^{t} \frac{1}{(1+s)^{\sigma+2}} U(s, X(s)) d s \\
& +2 \int_{0}^{t}(1+s)^{2 q-3} X(s) X(s-h) d s \\
& \leq \int_{0}^{t} \frac{1}{(1+s)^{2}} d s+\int_{0}^{t} \frac{1}{(1+s)^{\sigma+2}} U(s, X(s)) d s \\
& +\int_{0}^{t} \frac{1}{(1+s)} U(s, X(s)) d s \\
& +\int_{0}^{t}(1+s)^{2 q-3}|X(s-h)|^{2} d s \\
& =1-\frac{1}{1+t}+\int_{0}^{t} \frac{1}{(1+s)^{\sigma+2}} U(s, X(s)) d s+\int_{0}^{t} \frac{1}{(1+s)} U(s, X(s)) d s \\
& +\int_{0}^{t}(1+s)^{2 q-3}|X(s-h)|^{2} d s \\
& \leq|\psi|_{C}^{2}-\frac{1}{1+t}+\int_{0}^{t} \frac{1}{(1+s)^{\sigma+2}} U(s, X(s)) d s+\int_{0}^{t} \frac{1}{(1+s)} U(s, X(s)) d s \\
& +\int_{0}^{t}(1+s)^{2 q-3}|X(s-h)|^{2} d s \text {. }
\end{aligned}
$$


We now estimate the last integral by splitting it and using a change of variable

$$
\begin{aligned}
& \int_{0}^{t}(1+s)^{2 q-3}|X(s-h)|^{2} d s=|\psi|_{C}^{2} \int_{-h}^{0}(1+s+h)^{2 q-3} d s \\
& \quad+\int_{0}^{t-h}(1+r+h)^{2 q-3}|X(r)|^{2} d r \\
& \leq\left.|\psi|\right|_{C} ^{2}(1+h)^{2 q-3} \int_{-h}^{0} d s+(1+h)^{2 q-3} \int_{0}^{t-h} \frac{1}{1+s} U(s, X(s)) d s \\
& \leq h|\psi|_{C}^{2}(1+h)^{2 q-3}+(1+h)^{2 q-3} \int_{0}^{t} \frac{1}{1+s} U(s, X(s)) d s
\end{aligned}
$$

where we have used the inequality

$$
(1+r+h) \leq(1+r)(1+h), h>0, r \geq 0
$$

Then,

$$
\zeta_{\psi}(t)=|\psi|_{C}^{2}\left[h(1+h)^{2 q-3}+1\right], \quad \varphi(t)=\frac{1}{(1+t)^{\sigma+2}}+\frac{(1+h)^{2 q-3}+1}{(1+t)}, \quad \varrho(t)=-\frac{1}{1+t} .
$$

By some easy computation, we can check that

$$
\tau=0, \quad \nu=0, \quad \theta=\frac{(1+h)^{2 q-3}+1}{2} \quad \mu=\frac{\sigma}{2} \quad b=0 .
$$

Hence, by virtue of theorem 3.1 it follows

$$
\lim _{t \rightarrow+\infty} \sup \frac{\ln \left(|X(t)|-\left(\frac{1}{(1+t)\left(1+t^{2}\right)^{m}}\right)^{\frac{1}{q}}\right)}{\ln \left(1+t^{2}\right)} \leq-\left[2 q-2-\left(\frac{1}{2}\left((1+h)^{2 q-3}+1\right)+\frac{\sigma}{2}\right)\right], \quad \mathbb{P}-\text { a.s. }
$$

We have then proved practical asymptotic decay to zero with decay function $\lambda(t)=1+t^{2}$, $r(t)=\left(\frac{1}{(1+t)\left(1+t^{2}\right)^{m}}\right)^{\frac{1}{q}}$ and order at least

$$
\gamma=2 q-2-\left(\frac{1}{2}\left((1+h)^{2 q-3}+1\right)+\frac{\sigma}{2}\right)
$$

whenever

$$
q-1>\frac{\left(\frac{1}{2}\left((1+h)^{2 q-3}+1\right)+\frac{\sigma}{2}\right)}{2}
$$


Example 4.2. Consider the following semilinear stochastic partial differential equation:

$$
\left\{\begin{aligned}
d X(t, x) & =\left[2 \alpha \frac{\partial^{2}}{\partial x^{2}} X(t, x)+e^{\frac{-t}{2}} X(t-h, x)\right] d t+g(X(t, x))\left[\frac{X(t-h, x)}{1+|X(t, x)|}+e^{-\frac{3 t}{2}}\right] d W_{t}, \quad t \in[0, T], \\
X(t, x) & =\psi(t, x), \quad t \in[-h, 0], \quad x \in[0, \pi] \\
X(t, 0) & =X(t, \pi)=0, \quad t \geq 0
\end{aligned}\right.
$$

where $\alpha>0, h>0$ and $|\psi|_{C} \geq 1 ; g():. \mathbb{R} \rightarrow \mathbb{R}$ is a bounded Lipschitz continuous function such that $g(0) \neq 0$ and $|g(x)| \leq K, x \in \mathbb{R}, K>0$. Define $V=W_{0}^{1,2}([0, \pi]), H=L^{2}[0, \pi]$ and denote by ||.|| and |.| the norms in $V$ and $H$ respectively; by (.,.) the inner product in $H$. We can set this problem in our formulation by taking $A(t, u)(x)=2 \alpha \frac{\partial^{2} u(x)}{\partial x^{2}}$ for $u \in V, x \in[0, \pi]$; $F(t, \phi)(x)=e^{\frac{-t}{2}} \phi(-h)(x)$ and $G(t, \phi)(x)=g(\phi(0))\left[\frac{\phi(-h)(x)}{1+|\phi(0)(x)|}+e^{-\frac{3 t}{2}}\right]$, for $\phi \in C_{H}, t \geq 0$, $x \in[0, \pi]$. We will consider $U(t, y)=e^{2 t}|y|^{2}$ which immediately satisfies the whole assumptions required to apply Itô's formula. It is easy to check that, if we take $\xi(t)=\frac{1}{4 e^{2 t}}$, then

$$
\begin{aligned}
\int_{0}^{t} U_{s}^{\prime}(s, X(s)) d s & +\int_{0}^{t}<U_{x}^{\prime}(s, X(s)), A(s, X(s))+F\left(s, X_{s}\right)>d s \\
& +\frac{1}{2} \int_{0}^{t}\left(U_{x x}^{\prime \prime}(s, X(s)) G\left(s, X_{s}\right), G\left(s, X_{s}\right)\right) d s \\
& +\int_{0}^{t} \frac{1}{4 e^{2 s}}\left(U_{x}^{\prime}(s, X(s)), G\left(s, X_{s}\right)\right)^{2} d s \\
& \leq 2 \int_{0}^{t} U(s, X(s)) d s-\left.4 \alpha \int_{0}^{t} e^{2 s}|| X(s)\right|^{2} d s \\
& +2 \int_{0}^{t} e^{\frac{3}{2} s}|<X(s), X(s-h)>| d s+2 \int_{0}^{t} e^{2 s}|g(X(s))|^{2}|X(s-h)|^{2} d s \\
& +2 \int_{0}^{t} e^{2 s}|g(X(s))|^{2}|X(s-h)|^{2} \frac{|X(s)|^{2}}{(1+|X(s)|)^{2}} d s \\
& +2 \int_{0}^{t} e^{-s}|g(X(s))|^{2} d s+2 \int_{0}^{t} e^{-3 s}|g(X(s))|^{2} U(s, X(s)) d s \\
& \leq \int_{0}^{t}\left[2+2 K^{2} e^{-3 s}\right] U(s, X(s)) d s-4 \alpha \int_{0}^{t} e^{2 s}|| X(s)||^{2} d s \\
& +2 \int_{0}^{t} e^{\frac{3}{2} s}|X(s)||X(s-h)| d s+4 K^{2} \int_{0}^{t} e^{2 s}|X(s-h)|^{2} d s \\
& +2 K^{2} \int_{0}^{t} e^{-s} d s \\
& \leq \int_{0}^{t}\left[2+2 K^{2} e^{-3 s}\right] U(s, X(s)) d s+4 K^{2} I_{1}+I_{2}+I_{3}+2 K^{2} I_{4}, \\
&
\end{aligned}
$$


where $I_{1}=\int_{0}^{t} e^{2 s}|X(s-h)|^{2} d s, I_{2}=2 \int_{0}^{t} e^{\frac{3}{2} s}\left|X(s)\left\|X(s-h) \mid d s, I_{3}=-4 \alpha \int_{0}^{t} e^{2 s}\right\| X(s) \|^{2} d s\right.$ and $I_{4}=\int_{0}^{t} e^{-s} d s$.

$$
\begin{aligned}
I_{1} & \leq \int_{0}^{h}|X(s-h)|^{2} e^{2 s} d s+\int_{h}^{t} e^{2 s}|X(s-h)|^{2} d s \\
& \leq|\psi|_{C_{H}}^{2} \int_{-h}^{0} e^{2(h+u)} d u+\int_{0}^{t-h} e^{2(h+u)}|X(u)|^{2} d u \\
& \leq e^{2(h+t)}|\psi|_{C_{H}}^{2} \int_{-h}^{0} d u+\int_{0}^{t} e^{2(h+u)}|X(u)|^{2} d u \\
& \leq h e^{2(h+t)}|\psi|_{C_{H}}^{2}+e^{2 h} \int_{0}^{t} U(s, X(s)) d s .
\end{aligned}
$$

On the other hand,

$$
\begin{aligned}
I_{2} & \leq \int_{0}^{t} e^{\frac{3}{2} s}|X(s)|^{2} d s+\int_{0}^{t} e^{\frac{3}{2} s}|X(s-h)|^{2} d s \\
& \leq \int_{0}^{t} e^{-\frac{s}{2}} U(s, X(s)) d s+\int_{0}^{h} e^{\frac{3}{2} s}|X(s-h)|^{2} d s+\int_{h}^{t} e^{\frac{3}{2} s}|X(s-h)|^{2} d s \\
& \leq \int_{0}^{t} e^{-\frac{s}{2}} U(s, X(s)) d s+|\psi|_{C_{H}}^{2} \int_{-h}^{0} e^{\frac{3}{2}(u+h)} d u+\int_{0}^{t-h} e^{\frac{3}{2}(u+h)}|X(u)|^{2} d u \\
& \leq \int_{0}^{t} e^{-\frac{s}{2}} U(s, X(s)) d s+|\psi|_{C_{H}}^{2} e^{\frac{3}{2}(t+h)} \int_{-h}^{0} d u+\int_{0}^{t} e^{\frac{3}{2}(u+h)}|X(u)|^{2} d u \\
& \leq \int_{0}^{t} e^{-\frac{s}{2}} U(s, X(s)) d s+h|\psi|_{C_{H}}^{2} e^{\frac{3}{2}(t+h)}+e^{\frac{3}{2} h} \int_{0}^{t} e^{-\frac{s}{2}} U(s, X(s)) d s,
\end{aligned}
$$

and finally

$$
I_{3} \leq-4 \alpha \int_{0}^{t} U(s, X(s)) d s+3 K^{2} e^{-t}, \quad I_{4} \leq|\psi|_{C_{H}}^{2}-e^{-t}
$$


Therefore,

$$
\begin{aligned}
\int_{0}^{t} U_{s}^{\prime}(s, X(s)) d s & +\int_{0}^{t}<U_{x}^{\prime}(s, X(s)), A(s, X(s))+F\left(s, X_{s}\right)>d s \\
& +\frac{1}{2} \int_{0}^{t}\left(U_{x x}^{\prime \prime}(s, X(s)) G\left(s, X_{s}\right), G\left(s, X_{s}\right)\right) d s \\
& +\int_{0}^{t} \frac{1}{4 e^{2 s}}\left(U_{x}^{\prime}(s, X(s)), G\left(s, X_{s}\right)\right)^{2} d s \\
& \leq \int_{0}^{t}\left[4 K^{2} e^{2 h}+2+e^{-\frac{s}{2}}+e^{\frac{1}{2}(3 h-s)}-4 \alpha+2 K^{2} e^{-3 s}\right] U(s, X(s)) d s \\
& +|\psi|_{C_{H}}^{2}\left[4 K^{2} h e^{2(h+t)}+h e^{\frac{3}{2}(t+h)}+2 K^{2}\right]+K^{2} e^{-t} \\
& \leq \int_{0}^{t} \varphi(s) U(s, X(s)) d s+\zeta_{\psi}(t)+\varrho(t),
\end{aligned}
$$

where

$$
\varphi(t)=4 K^{2} e^{2 h}+2+e^{-\frac{t}{2}}+e^{\frac{1}{2}(3 h-t)}-4 \alpha+2 K^{2} e^{-3 t}, \quad \varrho(t)=K^{2} e^{-t}, \quad \lambda(t)=e^{t}
$$

and

$$
\zeta_{\psi}(t)=|\psi|_{C_{H}}^{2}\left[4 K^{2} h e^{2(h+t)}+h e^{\frac{3}{2}(t+h)}+2 K^{2}\right], \quad r(t)=K e^{-\frac{3}{2} t} .
$$

Therefore, constants in theorem 3.1 can be chosen as follows $\tau=0, \mu=\nu=m=q=2, \theta=2+4 K^{2} e^{2 h}-4 \alpha, b=0$. Hence we deduce that

$$
\lim _{t \rightarrow+\infty} \sup \frac{\ln \left(|X(t)|-K e^{-\frac{3}{2} t}\right)}{t} \leq-\left[2-\left(4+4 K^{2} e^{2 h}-4 \alpha\right)\right], \quad \mathbb{P}-\text { a.s. }
$$

If $2>4+4 K^{2} e^{2 h}-4 \alpha$, the solution is almost surely exponentially stable with decay function $\lambda(t)=e^{t}$ and order at least $\gamma=2-\left(4+4 K^{2} e^{2 h}-4 \alpha\right)$.

Remark 4.3. Although in our examples we have only considered constant delays, one can also analyze those cases in which the delay in the model is represented by some variable delay function or even in distributed form.

Acknowledgement. The research of T. Caraballo has been partially supported by FEDER and Ministerio de Economía y Competitividad grant \# MTM2011-22411 and Junta de Andalucía under Proyecto de Excelencia P12-FQM-1492 and \# FQM314 (Spain).

\section{References}

[1] B. Ben Hamed, I. Ellouze, M.A. Hammami, Practical uniform stability of nonlinear differential delay equations. Mediterranean Journal of Mathematics 8 (2011), no. 4, 603-616. 
[2] A. BenAbdallah, M. Dlala, M. A. Hammami, A new Lyapunov function for perturbed non linear systems, Systems and Control Letters 56 (3) (2007) 179-187.

[3] A. BenAbdallah, I. Ellouze and M. A. Hammami, Practical stability of nonlinear timevarying cascade systems, Journal of Dynamical and Control Systems, Vol. 15, No. 1, (2009) 4562 .

[4] M. J. Anabtawi, Practical Stability of Nonlinear Stochastic Hybrid Parabolic Systems of ItoType: Vector Lyapunov Functions Approach, Journal of Nonlinear Analysis: Real World Applications, Volume 12, Issue 3, (2011), 1386-1400.

[5] M. J. Anabtawi, Almost Sure Convergence Result of Stochastic Parabolic Partial Differential Equations, Journal of Dynamics of Continuous, Discrete and Impulsive Systems, Series A: Mathematical Analysis Volume 15, (2008) no. 1, 77-88.

[6] L. Arnold, A formula connecting sample and moment stability of linear stochastic systems, SIAM J. Appl. Math. 44 (1984), 793-802.

[7] T. Caraballo, M. J. Garrido-Atienza, J, Real, Existence and uniqueness of solutions to delay stochastic evolution equations, Stoch. Anal. Appl. 20 (6) (2002), 1225-1256.

[8] T. Caraballo, K. Liu, A. Truman, Stochastic functional partial differential equations: existence, uniqueness and asymptotic decay property, Proc. R. Soc. Lond. A 456 (2000), 17751802 .

[9] T. Caraballo, On the decay rate of solutions of non-autonomous differential systems, Electron. J. Diff. Eqns. 2001, 2001 (05), 1-17.

[10] T. Caraballo, Asymptotic exponential stability of stochastic partial differential equations with delay, Stochastics 33 (1990), 27-47.

[11] T. Caraballo, K. Liu, On exponential stability criteria of stochastic partial differential equations, Stochastic Proc. Appl. 83 (1999), 289-301.

[12] T. Caraballo, Maria J. Garrido-Atienza, José Real, Asymptotic Stability of Nonlinear Stochastic Evolution Equations, Stochastic Analysis and Applications, Vol. 21 (2003), No. 2, 301-327.

[13] T. Caraballo, Maria J. Garrido-Atienza, José Real, Stochastic stabilization of differential systems with general decate rate, Systems and Control Letters 48 (2003), 397-406.

[14] T. Caraballo, M. A. Hammami, L. Mchiri, Practical asymptotic stability of nonlinear stochastic evolution equations, Stochastic Analysis and Applications 32 (2014), 77-87.

[15] R. Z. Has'minskii, Stochastic Stability of Differential Equations, Sijthoff Noordhoff, 1980. 
[16] U. G. Haussmann, Asymptotic stability of the linear Itô equation in infinite dimensions, $J$. Math. Anal. Appl. 65 (1978), 219-235.

[17] H. K. Khalil, Nonlinear Systems, Mac-Millan, 2nd edition, 1996.

[18] R. Sh. Lipster, A. N. Shirayev, Theory of Martingales, Dordrecht. Kluwer Academic Publ Dordrecht, The Netherlands, (1989).

[19] K. Liu, X. R. Mao, Large time decay behavior of dynamical equations with random perturbation features, Stochastic Anal. Appl. 19 (2) (2001), 295-327.

[20] K. Liu, A. Truman, A stability of Stochastic Differential Equations In Infinite Dimensions, Monograph.

[21] X. Mao, Almost sure polynomial stability for a class of SDEs, Quart. J. Math. Oxford Ser. (2) 43 (1992), 339-348.

[22] X. Mao, Exponential Stability of Stochastic Differential Equations, Marcel Dekker, Inc.: New York, 1994.

[23] X. Mao, Stochastic Differential Equations and Applications, Ellis Horwood, Chichester, U.K, 1997.

[24] E. Pardoux, Équations aux Dérivées Partielles Stochastiques Non Linéaires Monotones, Thèse, Université Paris XI, 1975, 236.

[25] U. H. Thygesen, A survey of Lyapunov Techniques for Stochastic Differential Equations, IMM Technical Report n.c, 1997. 